\title{
LA MAGIA DEL TRIÁNGULO: CONVIVENCIA, CONFLICTO E INCLUSIÓN. UN ESTUDIO DE CASO A TRAVÉS DE UN PROGRAMA DE INTERVENCIÓN
}

\author{
Miguel Ángel Santos Guerra \\ Universidad de Málaga \\ Noemí Rodríguez Fernández \\ Alejandro Rodríguez Martín \\ María Verdeja Muñiz \\ Universidad de Oviedo
}

RESUMEN: El presente estudio se enmarca en la línea de trabajos que abogan por modelos integrados de convivencia positiva en los centros escolares orientados a potenciar sistemas participativos y aceptación de compromisos. El objetivo fue conocer la valoración de los participantes en un programa de mejora de la convivencia y poner en valor experiencias educativas de éxito que impulsan a la escuela como marco de convivencia pacífica y democrática. El diseño de investigación, basado en el estudio de casos, se planteó con una combinación de técnicas cualitativas (observación participante, entrevista en profundidad y grupos de discusión) y cuantitativas (cuestionario). La unidad de análisis es un programa de convivencia que se desarrolla en un Colegio Público del Municipio de Oviedo. En general, el clima de convivencia es bueno. Los conflictos suceden, principalmente, durante el recreo y en su mayor parte están motivados por las relaciones entre los chicos/as cuando juegan al fútbol. La metodología de las sesiones en el aula, basadas en la dinámica de grupos, favoreció un clima favorable para el aprendizaje y la buena relación entre los participantes. La implicación institucional y de toda la comunidad educativa apoyando el programa, fue un factor clave para el éxito del mismo.

PALABRAS CLAVE: convivencia, mediación, resolución de conflictos, participación, comunidad, inclusión.

\section{THE MAGIC OF THE TRIANGLE: COEXISTENCE, CONFLICT AND INCLUSION. A CASE STUDY THROUGH AN INTERVENTION PROGRAM}

ABSTRACT: This study is part of the line of work that opts for integrated models of positive coexistence in schools, aimed at promoting participatory systems and acceptance of commitments. The objective was to know how the 
participants assessed a program to improve coexistence and to value successful educational experiences that set the school as a framework of peaceful and democratic coexistence. The research design is based on case study and was posed with a combination of qualitative (participant observation, in-depth interview, and discussion groups) and quantitative (questionnaire) techniques. The unit of analysis was the coexistence program carried out in a Public School of the municipality of Oviedo. Overall, the climate of coexistence was good. The conflicts raised mainly in the playground, and for the most part was motivated by the relationships formed when playing football. The methodology of the classroom sessions, based on group dynamics, favoured a climate for learning and good relationship between participants. Institutional involvement, and that of the entire educational community, in supporting the program was a key factor in its success.

KEYWORDS: School coexistence, mediation, conflict resolution, participation, community, inclusion.

Recibido: 12/02/2021

Aceptado: 04/05/2021

Correspondencia: María Verdeja Muñiz, Facultad de Formación del Profesorado y Educación, Universidad de Oviedo, Campus de Llamaquique, C/ Aniceto Sela, s/n, 33005 Oviedo. Email: verdejamaria@uniovi.es

\section{INTRODUCCIÓN}

La educación y los sistemas educativos actuales se enfrentan a constantes cambios y nuevos desafíos. Mejorar la actividad educativa y favorecer la convivencia, el respeto hacia las demás personas, la igualdad de oportunidades y la no discriminación atendiendo a la diversidad del alumnado, son algunos de los retos educativos más importantes del momento.

El Informe a la UNESCO de la Comisión Internacional sobre la Educación para el Siglo XXI (Delors, 1996) ponía la mirada en la ampliación de los objetivos educativos más allá del estricto campo educativo y señalaba la necesidad de que los estudiantes aprendan a convivir en la escuela.

Diferentes autores (Ortega, Del Rey y Casas, 2015; Santos Guerra, 2015; Romera, Gómez-Ortiz y García-Fernández, 2016; Luengo, 2017) consideran que la escuela constituye uno de los espacios más importantes para construir la convivencia. Además, una de las principales finalidades de la educación es la de enseñar a convivir, porque como señala Santos Guerra (2003) no hay conocimiento útil si no sirve para formar a futuros ciudadanos/as capaces de convivir de forma pacífica.

En el contexto español destacamos el informe realizado por el Defensor del Pueblo (1999) en el que por primera vez se hacía referencia a una temática que trascendía el ámbito escolar, por lo que era necesario implicar a toda la sociedad en su conjunto. 
Desde entonces las administraciones educativas han puesto en marcha actuaciones para mejorar las relaciones entre iguales y se comienza a actuar en una doble vía; por un lado, de forma preventiva (Cowie, 2007; Fernández García, 2008) pero, también con actuaciones concretas cuando los problemas ya se han producido, así pues, destacamos programas como: Convivir es vivir de Carbonell (1997) o el proyecto Sevilla Anti-Violencia Escolar (SAVE) de Ortega (1997), programas que incorporan a estudiantes como mediadores (Torrego, 2006), programas que trabajan favoreciendo la ayuda entre iguales (Avilés et al., 2008; González Bellido, 2015) y programas que fomentan la participación de toda la comunidad educativa (Proyecto Atlántida, 2003).

Durante el periodo comprendido entre los años 1999-2006 se observan algunas mejoras, pero se hace necesario conocer los progresos, así pues, la oficina del Defensor del Pueblo elaboró un segundo informe (Defensor del Pueblo, 2007) a modo de seguimiento. En dicho informe se reflejaba que, a pesar de las mejoras, la problemática continúa presente en los centros, como así lo reflejan diferentes investigaciones (Jares, 2006; Díaz-Aguado et al., 2010; Zaitegi, 2010). A pesar de que se pude señalar que la escuela ya se ha puesto en marcha para mejorar la convivencia, la investigación educativa señala que es preciso incrementar, extender y hacer una evaluación y seguimiento de las medidas adoptadas.

\section{Marco legal para la mejora de la convivencia en los centros EDUCATIVOS}

En el marco normativo relacionado con actuaciones para mejorar la convivencia, hay que recordar que en el año 2006 se firma el Acuerdo entre el Ministerio de Educación y Ciencia y las organizaciones sindicales más representativas, contando con la implicación del profesorado, familias y alumnado. Está planteado para establecer un Plan de Actuación para la mejora de la convivencia que se concibe desde el diálogo y el consenso con las Comunidades Autónomas y la implicación de sus principales destinatarios: docentes, estudiantes y familias. Por ese motivo se emprenden actuaciones dirigidas hacia estos colectivos cuya participación y colaboración resultan imprescindibles para la mejora de la convivencia.

Por su parte la LOE (2006) en su preámbulo ponía el acento en la importancia que tiene la convivencia democrática, la prevención y resolución pacífica de conflictos. Así mismo, entre los principios de la educación que inspiraban dicha ley se hacía mención expresa a la educación para la convivencia, el respeto, la prevención de conflictos y la resolución pacífica de los mismos, así como para la no violencia en todos los ámbitos de la vida personal, familiar y social, y en especial en el del acoso escolar y ciberacoso.

En este contexto parece necesario que los centros escolares se constituyan como comunidades democráticas (Santos Guerra, 2009) donde los conflictos afloran, pero se abordan y se resuelven de forma pacífica.

También es importante recordar que durante el curso 2015-2016 se elaboró el Plan Estratégico de Convivencia Escolar coordinado por el Ministerio de Educación Cultura y Deporte, a través del Centro Nacional de Innovación e Investigación Educativa (MECD, 2016). Sus ejes fundamentales se basan en la educación inclusiva, la 
participación de la comunidad educativa, el aprendizaje de la convivencia, la educación en los sentimientos y en la amistad, la socialización preventiva de la violencia de género, la prevención de la violencia y la atención y cuidado del uso de las TIC.

La nueva ley orgánica de educación (LOMLOE, 2020) también establece un marco favorecedor para la convivencia ya que se plantea como una temática que se debe trabajar de forma transversal y en todas las etapas educativas, comenzando por la etapa de educación infantil.

\section{Aprender participando: programa de mejora de la convivencia Y LA EDUCACIÓN PARA LA PARTICIPACIÓN EN CENTROS EDUCATIVOS}

Como señalan Fierro y Carbajal (2019) en la década de los 90s la temática de la convivencia escolar cobró gran importancia y se presenta como una alternativa para la resolución de conflictos en los espacios educativos. En la actualidad la convivencia pasa de ser una temática a trabajar en los centros educativos sensibilizados a convertirse en un objetivo educativo que tiene un carácter institucional y que se incluye en el Proyecto Educativo del Centro. El abordaje de la convivencia adquiere un carácter preventivo y se desarrolla a través de acciones planificadas para trabajar la mediación, la resolución pacífica de conflictos, las habilidades sociales, etc.

En el 2000, el Movimiento Asturiano por la Paz (MASPAZ) ${ }^{1}$ pone en marcha el programa "Aprender participando: programa de mejora de la convivencia y educación para la participación en centros educativos" cuya finalidad es la formación de niños/as y adolescentes en participación social, desarrollando habilidades y actitudes necesarias para ver la realidad de una manera crítica, plantear alternativas y soluciones para mejorarla, organizarse y llegar al consenso, planificar acciones específicas y conjuntas, ejecutar pequeños proyectos teniendo en cuenta la permanente reflexión sobre la práctica y la evaluación constante.

En estos veinte años de andadura MASPAZ ha formado a numerosos mediadores en más de cuarenta centros educativos asentados en el Principado de Asturias. El programa se desarrolla anualmente atendiendo a los tres pilares básicos sobre los que se sustenta una verdadera participación social: el querer, el saber y el poder participar, como así se puede observar en el Gráfico 1.

Con relación al QUERER participar, la motivación es un elemento y necesario para iniciar el proceso, despertando en los alumnos/as intereses e inquietudes por colaborar en la mejora de la realidad en la que están inmersos. Para ello se realizan actividades de sensibilización con alumnado escolarizado en los cursos de $4^{\circ}, 5^{\circ}$ y $6^{\circ}$ de Educación Primaria y de $1^{\circ}$ y $2^{\circ}$ de Educación Secundaria Obligatoria. Estas sesiones tienen como objetivo conectar con el alumnado en cada centro donde se desarrolle el programa.

1. El Movimiento Asturiano por la Paz (MASPAZ, http://maspaz.org/) es una organización no gubernamental que nació en Asturias en 1991. Está integrada por personas que colaboran de manera independiente a la promoción y difusión de la paz. 
Gráfico 1. Pilares básicos de la participación

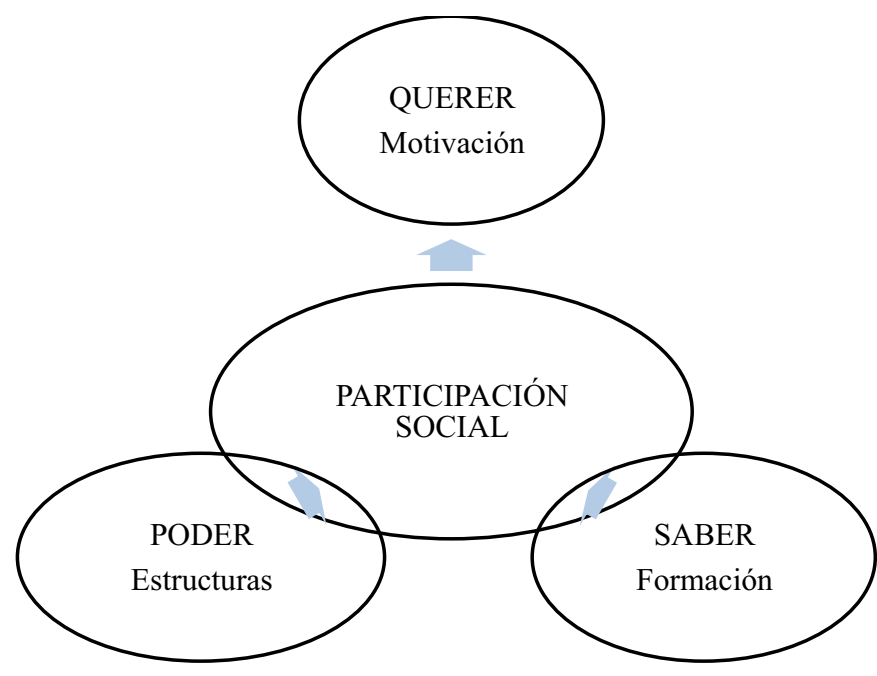

Con respecto al SABER participar, aunque es verdad que a participar se aprende participando, sabemos que para que este proceso se ejercite adecuadamente es preciso desarrollar en las personas una serie de habilidades, capacidades y valores que les permitan analizar de una manera critica la realidad en la que viven, relacionarse positivamente con los otros, extraer conclusiones comunes y planificar acciones conjuntas. Se realizan talleres dirigidos al alumnado voluntario para la participación y resolución pacífica de los conflictos.

Se forma a los participantes en estrategias de mejora de la convivencia entre las que se incluye la mediación escolar. Los estudiantes se integran en los equipos de convivencia y participan en la resolución de conflictos poniendo a disposición de sus compañeros un servicio de mediación escolar. Se plantean los siguientes requisitos previos a los centros educativos para iniciar el programa:

1) Interés y apoyo del equipo directivo de los centros.

2) El programa pasa a formar parte de la Programación General Anual (PGA).

3) El programa es difundido entre los miembros de claustro y las familias.

4) Se legitiman los procesos de resolución pacífica de conflictos dando protagonismo a los estudiantes voluntarios que se forman como mediadores

5) El equipo de mediación tiene referentes adultos en el centro y pasan a formar parte del equipo de convivencia.

6) El centro se compromete a buscar tiempos y espacios para la participación del alumnado.

Por último, el PODER participar, consiste en fortalecer y generar estructuras que hagan posible esta participación. Para ello se trabaja coordinadamente con el profe- 
sorado de los centros educativos, los equipos directivos y los órganos de gestión de los centros. El proyecto se realiza únicamente en centros educativos en los que existe una implicación del equipo directivo y de los equipos de convivencia. Todo ello lo mostramos resumido en el Gráfico 2.

Gráfico 2. Enfoque inclusivo-integrador en la resolución alternativa de conflictos

\begin{tabular}{|c|c|}
\hline & $\begin{array}{c}\text { SABER (CONCEPTUAL) } \\
\text { Conocimiento de nuestra realidad } \\
\text { Conocimiento de la realidad de los/as } \\
\text { otros/as }\end{array}$ \\
\hline & $\begin{array}{c}\text { HACER (PROCEDIMENTAL) } \\
\text { Transformar la realidad } \\
\text { Resolver problemas }\end{array}$ \\
\hline & $\begin{array}{c}\text { SER (ACTITUDINAL) } \\
\text { Responsabilidad } \\
\text { Solidaridad } \\
\text { Autonomía }\end{array}$ \\
\hline
\end{tabular}

Este programa se complementa con cursos de formación del profesorado sobre mejora de la convivencia y metodologías de trabajo con grupos, en colaboración con los Centros de Profesorado y Recursos del Principado de Asturias, a través de sus programas de formación en centros. Más de cincuenta docentes participan anualmente en dicha formación.

\section{Centros de Educación Primaria participantes en el programa, CURSO 2019-2020}

El programa se organiza en dos fases. En una primera fase (sensibilización) se realiza una sesión informativa con el alumnado. En el curso 2019-2020 los estudiantes que participaron en esta fase fueron un total de 222. En una segunda fase (formación) aquel alumnado que se muestra interesado se incorpora al programa. La selección de los centros participantes se realiza en base a los siguientes criterios: la trayectoria del centro, la implicación del equipo directivo y la participación de sus equipos de convivencia. En esta segunda fase en el curso escolar 2019-2020 se han incorporado al programa un total de 62 estudiantes que serán "aprendices de mediación".

\section{Planteamiento y diseño de la investigación}

La presente investigación está basada en el planteamiento de estudio de casos (Yin, 1989; Eisenhardt, 1989; Stake, 2005) poniendo especial atención en identificar 
y describir las formas de organización dirigidas a comprender las dinámicas presentes en contextos singulares, conociendo y comprendiendo las particularidades de la situación para comprender cómo funcionan las partes y las relaciones con el todo. Desde tales enfoques, se parte de la premisa de que es fundamental estudiar los fenómenos tal como aparecen en el momento de realizar el estudio y se apoyan en lo observado para comprender qué aspectos permiten alguna modificación que propicie una mejora. Dicha elección metodológica queda justificada por lo que Villareal (2017) denomina como un método de gran utilidad para explicar nuevos fenómenos e incrementar el conocimiento científico a partir de una investigación rigurosa que acontezca en unas condiciones adecuadas. Nuestra unidad de análisis es el programa de convivencia que se desarrolla en el Colegio Público del Municipio de Oviedo. El enfoque metodológico con el que abordamos esta investigación es una metodología mixta que combina técnicas de recogida de información cualitativas (entrevista en profundidad, observación participante, grupos de discusión) con técnicas cuantitativas, usando como principal instrumento el cuestionario.

\section{Características de la muestra}

El muestreo que realizamos es de tipo teórico o intencional. Total de centros educativos participantes en el Programa $(n=10)$. Total de alumnado sensibilizado en todos los centros educativos $(n=222)$. Total de alumnado que pasa a la fase de formación en el total de centros participantes $(n=62)$. El centro educativo en el que realizamos el estudio es un C.P. del Municipio de Oviedo $(n=1)$. En la Tabla 1 mostramos la distribución del alumnado participante en el estudio (fase de sensibilización y fase de formación).

Tabla 1. Distribución del alumnado participante en el estudio

\begin{tabular}{|c|c|c|c|c|}
\hline $\begin{array}{c}\text { Distribución del alumnado en las fases } \\
\text { de sensibilización y de formación }\end{array}$ & Chico & Chica & Total & $\%$ \\
\hline Alumnado sensibilizado en $4^{\circ}$ & 8 & 8 & 16 & $30 \%$ \\
\hline Alumnado sensibilizado en $5^{\circ}$ & 6 & 12 & 18 & $33 \%$ \\
\hline Alumnado sensibilizado en $6^{\circ}$ & 6 & 14 & 20 & $37 \%$ \\
\hline Total de alumnado sensibilizado & 20 & 34 & 54 & $100 \%$ \\
\hline Alumnado formado como mediador en $4^{\circ}$ & 3 & 4 & 7 & $35 \%$ \\
\hline Alumnado formado como mediador en $5^{\circ}$ & 2 & 5 & 7 & $35 \%$ \\
\hline Alumnado formado como mediador en $6^{\circ}$ & 3 & 3 & 6 & $30 \%$ \\
\hline Total de alumnado formado como mediador & 8 & 12 & 20 & $100 \%$ \\
\hline
\end{tabular}

En la fase de sensibilización realizada en dicho Centro utilizamos el cuestionario con alumnado de $4^{\circ}, 5^{\circ}$ y $6^{\circ}$ curso de Educación Primaria. En dicha fase participaron un total de 54 estudiantes distribuidos de la manera que mostramos en el Gráfico 3. Alumnado participante en la fase de sensibilización. 
Gráfico 3. Alumnado participante en la fase de sensibilización

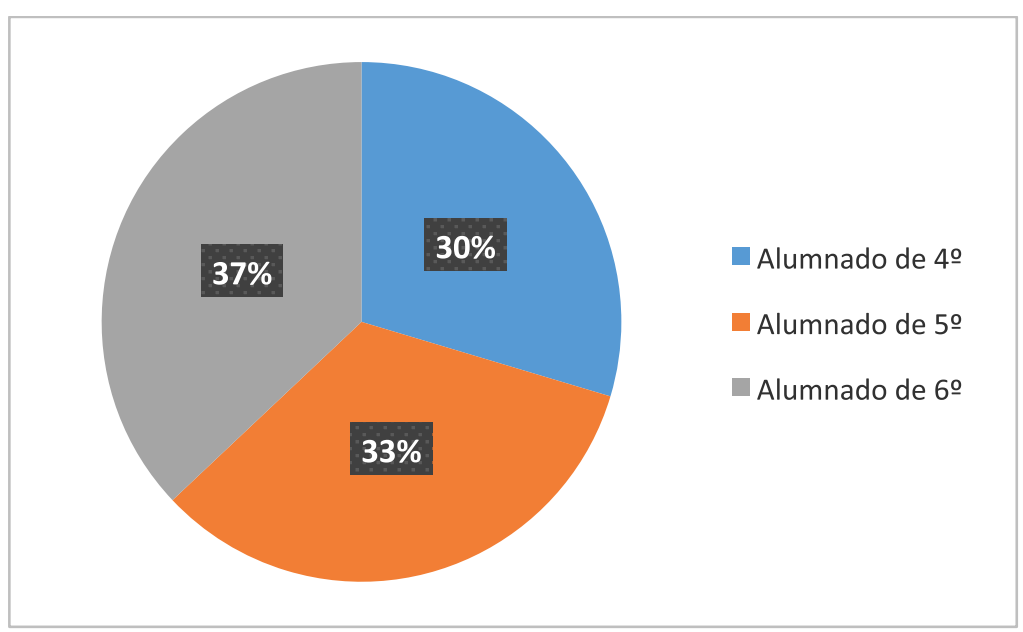

En la siguiente fase, formación, participaron un total de 20 estudiantes, distribuidos como aparece reflejado en el Gráfico 4. Alumnado formado como mediador.

Gráfico 4. Alumnado formado como mediador

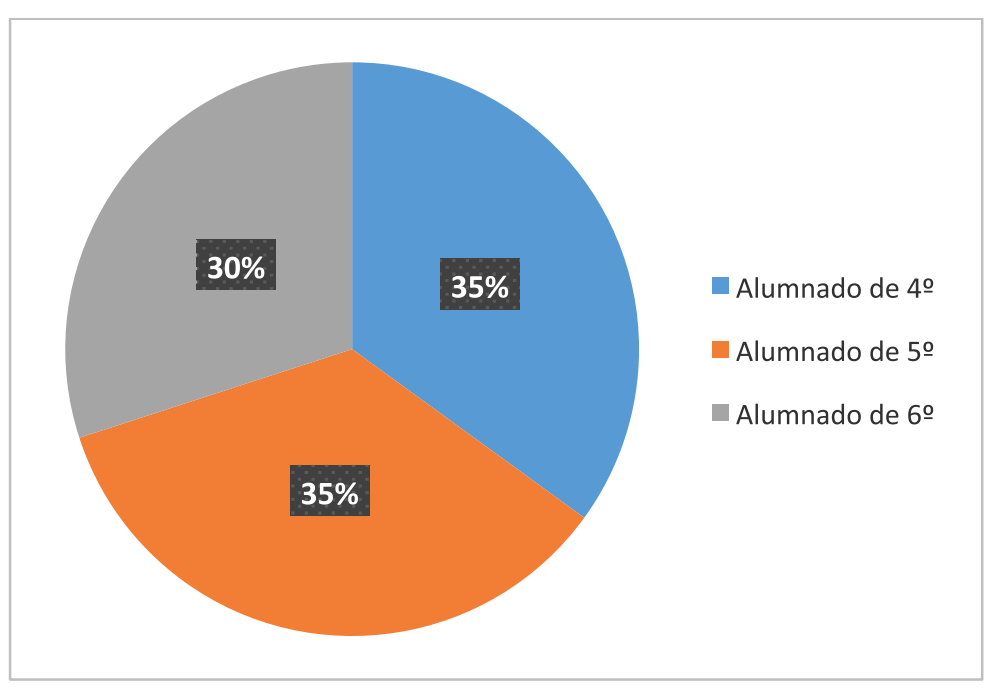

\section{INSTRUMENTOS Y PROCEDIMIENTO PARA LA RECOGIDA DE INFORMACIÓN}

La recogida de información se desarrolló en diferentes momentos de la investigación y con diferentes propósitos, tal y como se resume en la Tabla 2. 
Tabla 2. Técnicas e instrumentos de recogida de información

\begin{tabular}{|c|c|l|}
\hline $\begin{array}{c}\text { Técnicas e instrumentos } \\
\text { de recogida de información }\end{array}$ & $\begin{array}{c}\text { Momento } \\
\text { de } \\
\text { aplicación }\end{array}$ & \multicolumn{1}{|c|}{ Objetivo(s) } \\
\hline $\begin{array}{c}\text { Entrevista en profundidad a } \\
\text { la directora }\end{array}$ & Inicial & $\begin{array}{l}\text { Conocer la percepción de la directora sobre los } \\
\text { problemas de convivencia y su opinión acerca } \\
\text { del programa. }\end{array}$ \\
\hline $\begin{array}{c}\text { Observación participante en } \\
\text { el aula }\end{array}$ & Proceso & $\begin{array}{l}\text { Observar el desarrollo de las sesiones y la } \\
\text { metodología de trabajo en el aula. }\end{array}$ \\
\cline { 2 - 4 } $\begin{array}{c}\text { Cuestionario al alumnado } \\
\text { Observar la dinámica de las sesiones y cómo } \\
\text { trabaja el alumnado. }\end{array}$ \\
\hline $\begin{array}{c}\text { Grupos de discusión con el } \\
\text { alumnado del programa }\end{array}$ & Inicial & $\begin{array}{l}\text { Conocer la percepción que tienen los } \\
\text { alumnos/as del conflicto, así como los estilos } \\
\text { predominantes de afrontarlos. }\end{array}$ \\
\hline
\end{tabular}

Como así comentamos, en la fase previa (sensibilización) utilizamos el cuestionario. Este instrumento lo empleamos con todo el alumnado y tiene como principal objetivo conocer la percepción del alumnado acerca de la convivencia, así como las formas en que los chicos/as resuelven los conflictos. El cuestionario ha sido elaborado ad hoc y esto es debido a dos cuestiones fundamentales. Por un lado, la necesidad de ajustarnos a las peculiaridades y características del contexto educativo asturiano y, por otra parte, la necesidad de introducir preguntas abiertas relacionadas con los objetivos de nuestra investigación. El cuestionario es de tipo mixto y contiene preguntas cerradas con opciones de respuesta con una escala tipo Likert y también preguntas abiertas. Para su elaboración tomamos como referencia la propuesta de Ortega y del Rey (2003).

En cuanto a las técnicas cualitativas empleadas, una de ellas fue la entrevista en profundidad. Dicha técnica la utilizamos con la intención de comprender peculiaridades y características de una de las informantes clave: la directora del colegio quien, además, es la coordinadora en el centro del equipo de mediación. Para su diseño y elaboración tuvimos en cuenta dimensiones de análisis relacionadas con los objetivos de investigación. Las preguntas de la entrevista se plantearon en torno a tres dimensiones de análisis: 1) la percepción acerca de la convivencia en el centro y de los principales problemas de convivencia 2) espacios en los que se producen más problemas de convivencia 3) influencia del programa en la mejora de la convivencia. La entrevista, una vez realizada y transcrita, fue enviada a la directora para su revisión y validación. Una vez revisada por la directora, procedimos a realizar el análisis de contenido.

Otra de las técnicas cualitativas de recogida de información son los grupos de discusión con el alumnado. Consideramos que es necesario contar con la voz de los chicos/as y conocer, de primera mano, sus opiniones. El empleo de esta técnica tiene tres objetivos fundamentales: en primer lugar, la oportunidad de escuchar las opiniones de los protagonistas del proceso de enseñanza aprendizaje: el alumnado, en segundo lugar, nos brindaría la posibilidad de completar la información recopilada a partir de otras técnicas y, por último, nos ayudaría a contrastar la información a través de procesos de triangulación de fuentes y de técnicas (Denzin y Lincoln, 2000). 
Las preguntas de los grupos de discusión con el alumnado se estructuraron en torno a tres cuestiones clave: 1) conocer los espacios en los que se producen los conflictos 2) conocer las relaciones que se entretejen entre los chicos/as cuando juegan al fútbol y 3) observar las actividades que más les motivan del programa. La utilización de diferentes técnicas ha contribuido a un fin básico: la triangulación de fuentes y técnicas y, por lo tanto, asegurar la propia credibilidad de la información recogida, además, nos brinda la posibilidad de recoger información con carácter plural, diverso y holístico (Creswell, 2010).

Otra de las técnicas cualitativas es la observación participante. Es necesario recordar que una particularidad de esta iniciativa es que el programa para la mejora de la convivencia forma parte de la programación de actividades extraescolares del centro. Así pues, las sesiones tienen lugar un día a la semana (los lunes) y se desarrollan en el horario de extraescolares. Las observaciones en el aula están orientadas a conocer la metodología de trabajo. Para ello contamos con el diario de campo en el que se registraron anotaciones sobre lo que acontece en el desarrollo de las sesiones. Durante el proceso de observación (17 sesiones) estuvimos especialmente atentos a recoger información y a escuchar la voz del alumnado. Para completar las observaciones se contó con la partición de un observador externo. La persona que realizó la observación externa devolvió las observaciones recogidas y las compartió con la persona que desarrollaba la sesión en el aula para ser contrastadas. De esta forma la triangulación de técnicas y perspectivas permitió contrastar la información y enriquecer la investigación.

\section{La ética en el CAMPo}

El ingreso en un escenario generalmente implica una especie de pacto: la seguridad implícita o explícita de que no se desea violar la privacidad o confidencialidad de los informantes, ni exponerlos a prejuicios ni interferir en sus actividades (Taylor y Bogdan, 1994, p. 94). Teniendo en cuenta la importancia de los valores éticos en la investigación tuvimos en cuenta los siguientes principios: 1) respetar y garantizar el anonimato y confidencialidad de la información recogida, 2) tratar con sumo cuidado la "invasión de la privacidad" de los participantes, 3) solicitar consentimiento a los informantes en caso de tener que efectuar alguna grabación en audio y respetar siempre sus decisiones, 4) adquirir compromisos con los participantes con la idea de compartir las informaciones derivadas de la investigación, 5) adquirir compromisos para la negociación del informe y la comunicación de los resultados, 6) mostrar las ventajas de la realización del estudio disipando posibles miedos de cara a sentirse "cuestionados".

En cuanto al procedimiento para realizar el consentimiento informado de las familias, fue el siguiente: Lo primero de todo, cabe recordar que el programa forma parte de las actividades extraescolares que oferta el Centro, por lo tanto, las familias saben que sus hijos/as se encuentran matriculados en dicha actividad extraescolar. Con motivo de nuestra investigación, desde la dirección del centro, se envió una carta a las familias comunicando que un grupo de investigadores íbamos a realizar un estudio y ello conllevaría acceder a las aulas, observar las sesiones, realizar grupos de discusión, etc. Las familias o tutores legales firmaron el consentimiento informado para la participación del alumno/a en la investigación. Asimismo, también se lo comunicamos al alumnado y dio su consentimiento que quedó recogido por escrito en 
la parte inicial del instrumento. Cabe destacar que siempre se mostró entusiasmado e ilusionado por saber que estaban participando en una investigación.

\section{ANÁLISIS DE LA INFORMACIÓN}

Una vez que recogimos la información procedemos a organizar y transformar la misma en datos manejables para proceder a su interpretación. El análisis de la información recogida a partir de las técnicas cualitativas se ha realizado a través del programa informático de análisis cualitativo ATLAS.ti 8, lanzado inicialmente por Thomas Mühr (Universidad de Berlín) en 1993². Este software es considerado por varios evaluadores (Lewis, 1998; Weitzman, 2000; Klüber, 2014) como el mejor, más completo y fácil de todos para el apoyo a la comprensión e interpretación de los datos cualitativos. Con relación al análisis de la información obtenida a partir de las técnicas cuantitativas (cuestionario) el análisis se ha realizado con el programa estadístico SPSS 24.

\section{Resultados}

\subsection{Percepción positiva del clima de convivencia}

En general, el alumnado participante en la fase previa (sensibilización) tiene una percepción positiva del clima de convivencia, como así representamos en el Gráfico 5.

Gráfico 5. Percepción del clima de convivencia

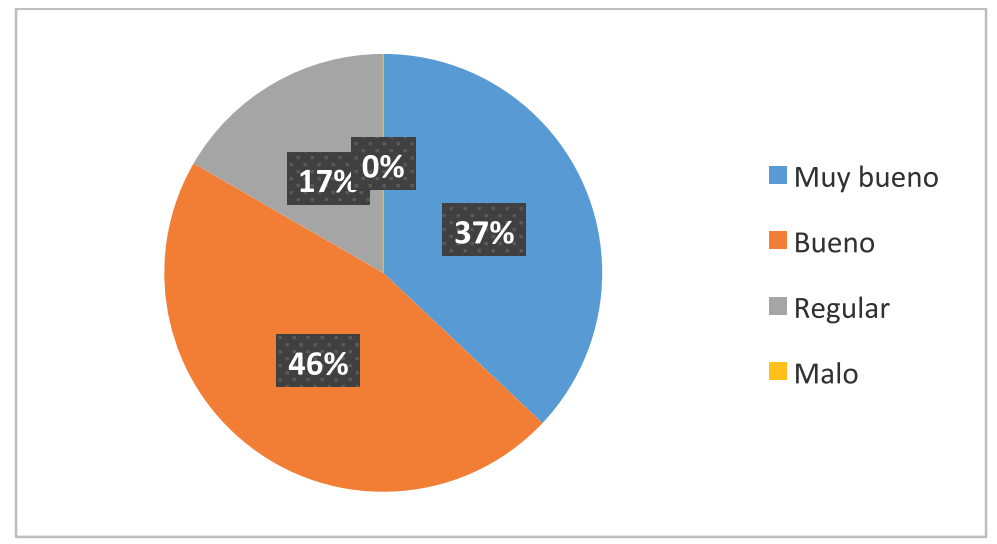

Esto, a su vez, está en coherencia con la entrevista realizada a la directora, que también mostró una percepción positiva del clima de convivencia. Reconoce que, como es natural, existen conflictos, pero menciona que éstos son minoritarios. También comenta que el programa ha contribuido a mejorar la convivencia.

2. ATLAS.ti (Scientific Software Development $\mathrm{GmbH}$ ) es un potente conjunto de herramientas para el análisis cualitativo de grandes cuerpos de datos textuales, gráficos y de vídeo. Más información disponible en: https://atlasti.com/ 
Los conflictos, aunque existen, son minoritarios, pero creo que es gracias a la prevención de estos. El programa nos ha servido para conseguirla (entrevista a la directora).

\subsection{Los principales conflictos se suelen dar en el tiempo del recreo y en su mayor parte están motivados por las relaciones humanas que tejen jugando al fútbol}

El hecho de que exista una percepción positiva del clima de convivencia no quita para que los alumnos/as reconozcan que existen problemas de convivencia. El análisis de la información recogida a través de las diferentes técnicas (cuestionarios, grupos de discusión y entrevista con la directora) permite constatar que uno de los principales espacios en los que tienen lugar más conflictos es en el recreo. Las relaciones humanas que se tejen cuando los chicos/as juegan al fútbol se ha convertido en uno de los principales focos de conflictos, como así aparece representado en el Gráfico 6.

Gráfico 6. Principales motivos de conflictos

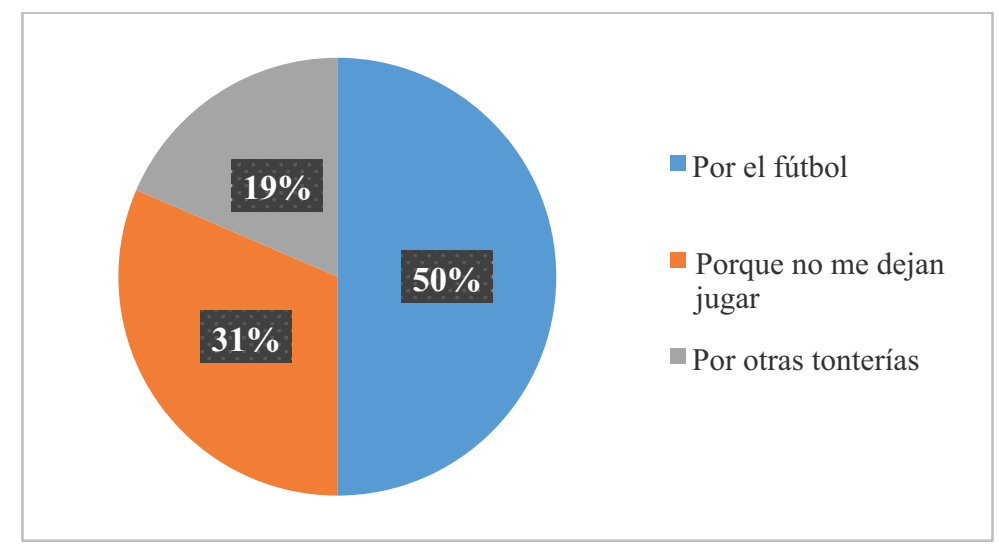

Para profundizar más en los conflictos que se generan cuando los chicos/as juegan al fútbol, tenemos la oportunidad de preguntar a los alumnos/as en el grupo de discusión. En un primer momento los alumnos/as señalan que pueden jugar al futbol en el patio del recreo todos los días, excepto los viernes. Es una norma propuesta por el centro, debido a la cantidad de desencuentros que se producían. Para resolver este problema el centro ha organizado los tiempos en que los distintos grupos pueden jugar al fútbol. Tal decisión ha contribuido a mejorar la problemática. Ahora el problema ya no es tanto por el espacio, sino que hay situaciones que son foco de conflictos:

Cada día un grupo puede jugar al fútbol, por ejemplo, un lunes es para los de $3^{\circ}$, el martes es para los de $4^{\circ}$, el miércoles para los de $5^{\circ}$, los jueves es para los de $6^{\circ}$ y el viernes no hay fútbol. Hay veces que, por ejemplo, uno le metió un balonazo a otro y se empiezan a enfadar y uno se chiva (grupo de discusión).

Esta cuestión también aparece en la entrevista a la directora. Si bien la directora del centro manifiesta que la convivencia en el centro, en general, es buena, tam- 
bién menciona que el patio es uno de los espacios donde se producen más conflictos y muchos de ellos tiene que ver con las relaciones que se producen cuando juegan al fútbol:

Generalmente es en el patio, en el juego libre es donde se producen más conflictos. Los conflictos tienen que ver, generalmente, por el balón, por las diferencias en el juego, etc. (entrevista a la directora).

\subsection{Una de las formas más frecuentes para resolver los conflictos es a través del diálogo}

En cuanto a las formas en que los alumnos/as prefieren resolver los conflictos, destaca el diálogo como una de las primeras formas que señalan para resolver el conflicto. Aunque también utilizan otras estrategias, por ejemplo, tratan de evitar que dicho conflicto se produzca, como así aparece representado en el Gráfico 7.

Gráfico 7. Principales formas de resolución de conflictos

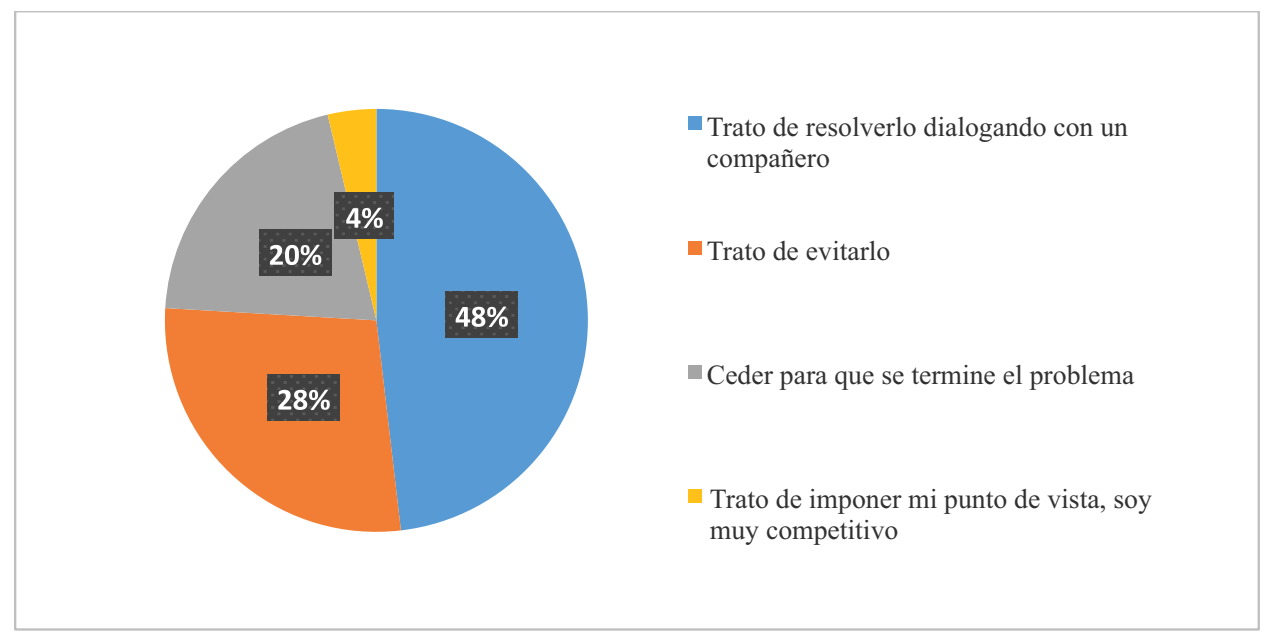

En la entrevista realizada a la directora, también se refleja que una de las principales formas de resolución de conflictos es a través del diálogo, aunque también reconoce que en ocasiones es necesaria la ayuda de un adulto:

Los conflictos que se pueden producir se resuelven generalmente dialogando, aunque a veces es necesaria la ayuda de un adulto (entrevista a la directora).

\subsection{Valoración positiva del programa de convivencia}

En general la valoración del programa de convivencia es muy positiva y en este centro es el quito año que se desarrolla por lo que se van observando algunos avances: 
Este es el quinto curso que se realiza el programa. La valoración es muy positiva, tanto para el alumnado como para las familias. Solo hay que pensar que cada año el número de alumnos participantes aumenta. Y muchas veces el papel de mediador les ayuda a solucionar sus propios conflictos con otros compañeros, a empatizar con los demás (entrevista a la directora).

\subsection{La metodología basada en la dinámica de grupos favorece un clima para el aprendizaje y la buena relación entre los participantes}

Uno de los éxitos del programa tiene que ver con la diversidad de actividades que se plantean, cada día una diferente, y otra cuestión muy importante es la metodología, basada en la dinámica de grupos. Las actividades están plenamente pensadas para el alumnado disfrute $y$, al mismo tiempo, aprenda:

La dinámica que estaba preparada les encantó. Se han volcado trabajando en los grupos, incluso se podía ver cómo había niños que habían acabado su tarea en un grupo, ayudaban a los niños/as de otro grupo (nota del diario de campo).

Nos gustan mucho todas las actividades. Soy muy divertidas y aprendemos a respetarnos. A mí me gusto mucho el día que grabamos una canción que estaba basada en la mediación (grupo de discusión).

A mí la actividad que más me gustó es la de las pancartas de los animales, sobre los estilos de afrontar los conflictos (grupo de discusión).

\subsection{Necesidad de fortalecer y trabajar las habilidades sociales del alumnado}

Una de las prioridades del centro educativo para implementar el programa, tiene que ver con la necesidad de fortalecer y trabajar habilidades sociales, así como la necesidad de fomentar hábitos de empatía entre el alumnado, reforzar lazos de convivencia y aprender a ponerse en el lugar del otro:

Uno de los principales objetivos que pretendemos alcanzar con este programa es conseguir un clima de centro armónico, en el que se puedan resolver los conflictos de manera pacífica y cooperativa. Desarrollar en las personas una serie de valores, capacidades y habilidades que le permitan analizar de manera crítica la realidad en que viven, y por último generar y fortalecer estructuras que posibiliten la participación, trabajando coordinadamente con la Comunidad Educativa, con un compromiso de formación en el ámbito de la mejora de la convivencia escolar (entrevista a la directora).

\subsection{Importancia de la implicación de toda la comunidad educativa}

Una de las claves de éxito del programa tiene que ver con el respaldo institucional y de toda la comunidad educativa. La participación sólo puede entenderse desde una vertiente inclusiva que sea capaz de dar respuesta a toda la comunidad educativa en su conjunto:

Se trata de un programa aprobado por el Claustro que forma parte de la Programación General Anual. También cuenta con el respaldo y la aprobación de las familias, así 
pues, se trata de un programa en el que está implicada toda la comunidad educativa (entrevista a la directora).

\subsection{Algunas propuestas de mejora del programa de convivencia}

Una de las claves de éxito del programa tiene que ver la formación de alumnos/ as mediadores que una vez han pasado por el proceso de formación en el programa, actúan como mediadores ante posibles conflictos. En ese sentido los estudiantes desarrollan bien su nuevo papel como mediadores, sin embargo, se hace necesario dar un paso más y complementar la figura del mediador/a con la de dinamizador/a, como así reclama la directora:

Nos gustaría que la formación de mediador se complementara con la de dinamizador, en los momentos de patio los mediadores podrían realizar tareas de dinámicas de patio, proponiendo juegos alternativos y creando momentos de juego y convivencia (entrevista a la directora).

\subsection{Algunas ideas que proponen los alumnos/as para mejorar la convivencia}

En el cuestionario había preguntas abiertas y en una de ellas se preguntaba sobre algunas ideas para mejorar la convivencia. El fútbol ocupa un importante lugar, aunque los alumnos/as también señalan algunas propuestas que tienen que ver con las formas de relacionarse entre ellos. Aquí destacamos las siguientes:

Hacer juegos todo el colegio juntos. Tener menos futbol. Quitar el fútbol y hablar si hay algún problema. Hacer un día a la semana un juego de convivencia. Pues que se acabe el fútbol y así la gente conviviría mejor. Que el fútbol sea más controlado. Que dejen jugar a los demás y no haya peleas ni conflictos. Más días sin fútbol (grupo de discusión).

\section{Algunas limitaciones del estudio}

Es necesario reconocer que este estudio de caso tiene algunas limitaciones. Por una parte, cabe recordar que el muestreo es de tipo teórico y ello implica un sesgo claro en los resultados de la investigación, así pues, hay que tener en cuenta que los resultados derivados se reducen a un contexto específico y entendemos que no pueden ser generalizables a otros contextos, lo que no quita para otorgar valor a los mismos y considerarlos como unos resultados que ponen de manifiesto el valor que la comunidad educativa de un Centro otorga a un programa de mejora de la convivencia. También es necesario reconocer que la muestra es reducida, si bien, al tratarse de un estudio de caso, hemos profundizado en la realidad de un contexto educativo concreto. Sin embargo, estos aspectos constituyen en sí mismos líneas de mejora en las que seguir explorando y que, en resumen, tienen un denominador común: la implicación de la comunidad educativa y el apoyo institucional a un programa de mejora de la convivencia donde la apuesta firme del equipo directivo es fundamental en los procesos de cambio y de mejora de la convivencia. 


\section{Discusıón}

Aprender participando: programa de mejora de la convivencia y educación para la participación en centros educativos, es un programa que, en la línea de la prevención, desarrolla una propuesta orientada a la mejora de la convivencia a través de la formación de alumnos/as mediadores. Al igual que otras iniciativas (García-Raga et al., 2016; Torrego, 2017; Pinto da Costa et al., 2020; Galindo y Sanahuja, 2021) la mediación para la resolución de conflictos representa una estrategia muy favorable para trabajar con el alumnado y tiene múltiples beneficios.

El programa incluye una serie de sesiones en las que se forma al alumnado en estrategias de mejora de la convivencia y participación en la resolución alternativa de conflictos, atendiendo al enfoque integrado e inclusivo. Asimismo, el planteamiento y dinámica de las sesiones favorece la participación y la implicación del alumnado. Estas dinámicas empoderan al alumnado haciéndole conocedor de sus derechos y deberes desde posiciones activas, participativas y críticas, siendo éste capaz de manejar procesos que se relacionan con la convivencia escolar y los conflictos. La metodología utilizada, basada en la dinámica de grupos, favorece el desarrollo de sesiones dinámicas y de gran interés y motivación para el alumnado, además, las actividades realizadas contribuyen a crear confianza y lograr un buen clima de interrelaciones entre los participantes mostrando el valor del trabajo en equipo y planteando críticas constructivas orientadas a la resolución de los dilemas planteados. Se atiende al modelo pedagógico integrador y generador de conciencia social, estableciendo principios pedagógicos que permitan el establecimiento de relaciones positivas y apoyado por metodologías que favorecen el aprendizaje significativo.

Se trata de un programa que también contempla el ámbito de las emociones con sesiones que permiten a los alumnos/as conocerse mejor a sí mismos, conocer el punto de vista del otro, mejorar el respeto hacia uno mismo y hacia los demás, etc. y, al igual que otras experiencias educativas (Gómez-Ortiz, et al., 2017) trabajar las emociones, favorece el desarrollo de las competencias sociales y emocionales de los alumnos/as.

En cuanto a las principales estrategias para abordar los problemas de convivencia, parece que la mediación cobra cada vez más relevancia. Al mismo tiempo, y partiendo de una perspectiva positiva de conflicto (Cascón, 2000) la mediación para la resolución de conflictos, el diálogo, la formación de alumnos/as mediadores y la ayuda entre iguales son estrategias clave para construir la convivencia. En coherencia con los modelos integrales de convivencia escolar (Torrego, 2006) la propuesta que aquí se observa, desarrollada por MASPAZ, está en línea de la perspectiva positiva del conflicto, que alude a este no solo como algo natural e inherente a todo tipo de organizaciones y a la vida misma, sino que, además, se configura como un elemento necesario para el cambio social y la mejora de las instituciones educativas. Precisamente, los resultados obtenidos en esta investigación ponen de manifiesto la necesidad de implementar y evaluar programas de intervención y mejora de la convivencia. Una de las claves del éxito del programa es la implicación institucional. Como señala San Fabián (2011) la intervención educativa no puede obviar lo organizativo. Así, cuando la comunidad educativa se siente identificada con el programa, que tiene un carácter institucional y forma parte de la vida del centro, es un factor clave para el éxito del mismo y en este caso la mejora de la convivencia. 


\section{REFERENCIAS BIBLIOGRÁFICAS}

ATLAS.ti (2020). The Qualitative Data Analysis \& Research Software - ATLAS.ti. Scientific Software Development, GmbH. Berlín. https://atlasti.com/

Avilés, J. M., Torres, N. y Vián, M. V. (2008). Equipos de ayuda, maltrato entre iguales y convivencia escolar. Revista de Investigación Psicoeducativa, 6, 357-376.

Carbonell, J. L. (Coord.) (1997). Convivir es vivir. Madrid: Defensor del Menor.

Cascón, F. (2000). La mediación. Cuadernos de Pedagogía, 287, 72-76.

Cowie, H. (2007). Trabajar con las relaciones para mejorar la convivencia, IDEA-La Mancha. Revista de Educación de Castilla La Mancha, 4, 101-106.

Creswell, J. W. (2010). Mapping the developing landscape of mixed methods research. En Handbook of mixed methods in social and behavioral research (2nd Ed, pp. 45-68). Thousand Oaks, CA: Sage.

Delors, J. (1996). La educación encierra un tesoro. Informe a la UNESCO de la Comisión internacional sobre la educación para el siglo XXI. España: UNESCO.

Defensor del Pueblo. (2000). Informe sobre violencia escolar: el maltrato entre iguales en la Educación Secundaria Obligatoria. Madrid. Oficina del Defensor del Pueblo.

Defensor del Pueblo. (2007). Violencia escolar: el maltrato entre iguales en la educación secundaria obligatoria 1999-2006 (Nuevo estudio y actualización del informe 2000). Madrid: Publicaciones de la Oficina del Defensor del Pueblo.

Denzin, N. K. y Lincoln, Y. S. (Eds.) (2000). Handbook of Qualitative Research. Thousand Oaks: Sage.

Díaz-Aguado, M. J., Martínez, R. y Martín, J. (2010). Estudio Estatal sobre la Convivencia. Escolar en la Educación Secundaria Obligatoria. Madrid: Ministerio de Educación y Observatorio Estatal de la Convivencia.

Eisenhardt, K. M. (1989). Building Theories from Case Study Research. Academy of Management Review, 14(4), 532-550.

Fernández García, I. (2008). Los programas de ayuda para la mejora de la convivencia en instituciones, Bordón. Revista de Pedagogía, 60(4), 137-150.

Fierro, C. y Carbajal, P. (2019). Convivencia escolar: Una revisión del concepto. Psicoperspectivas, 18(1), 1-14.

Galindo, E. I. y Sanahuja, A. (2021). Implantación de un proyecto de mediación entre iguales: hacia una mejora de la convivencia en el contexto educativo. Márgenes, Revista de Educación de la Universidad de Málaga, 2(1), 130-147. https://doi. org/10.24310/mgnmar.v2i1.8459

García-Raga, L., Chiva, I., Moral, A. y Ramos, G. (2016). Fortalezas y debilidades de la mediación escolar desde la perspectiva del alumnado de educación secundaria. Pedagogía Social. Revista Interuniversitaria, 28, 203-215.

Gómez-Ortiz, O., Romera, E. M. y Ortega, R. (2017). La competencia para gestionar las emociones y la vida social y su relación con el fenómeno del acoso y la convivencia escolar. Revista Interuniversitaria de Formación del Profesorado, 88(31.1), 27-38.

González Bellido, A. (2015). Programa TEI Tutoría Entre Iguales. Innovación Educativa, 25, 17-32. 
Jares, X. R. (2006). Conflicto y convivencia en los centros educativos de secundaria. Revista de Educación, 339, 467-491.

Klüber, T. (2014). Atlas.ti as a tool for analysis of qualitative research according phenomenological approach. ETD: Educaçao Temática Digital, 16(1) Ejemplar dedicado a: Pesquisa, desenvolvimento e formação na educação, 5-23. https://doi. org/10.20396/etd.v16i1.1326

Lewis, R. B. (1998). ATLAS/ti and NUD.IST: A Comparative Review of Two Leading Qualitative Data Analysis Packages. CAM Journal, 10(3), 41-47. https://doi.org/10 .1177/1525822X980100030101

Ley Orgánica 2/2006, de 3 de mayo, de Educación. Boletín Oficial del Estado. Madrid, 4 de mayo de 2006, núm. 106, pp. 17158-17207.

Ley Orgánica 3/2020, de 29 de diciembre, por la que se modifica la Ley Orgánica 2/2006, de 3 de mayo, de Educación. Boletín Oficial del Estado. Madrid, 30 de diciembre de 2020, núm. 340, pp. 122868-122953.

Luengo, J. A. (2017). Promover la convivencia en los centros educativos: El protagonismo del alumno. Revista de Estudios de Juventud, 115, 97-114.

Ministerio de Educación Cultura y Deporte (2016). Plan estratégico de convivencia escolar. Confiar en la fuerza de la educación. Madrid: Secretaría General Técnica. Madrid: Centro de Publicaciones del Ministerio de Educación, Cultura y Deporte.

Ortega, R. (1997). El Proyecto Sevilla anti-violencia escolar. Un modelo de intervención preventiva contra los malos tratos entre iguales. Revista de Educación, 313, 143-158.

Ortega, R. y Del Rey, R. (2003). La violencia escolar. Estrategias de prevención. Barcelona: Graó.

Ortega, R., Del Rey, R. y Casas, J. A. (2015). La convivencia escolar: Clave en la predicción del bullying. Revista Iberoamericana de Evaluación Educativa, 6(2), 91-102.

Pinto da Costa, E., De Oliveira Martins, A., Torrego, J. C. y Oliveira e Sá, S. (2020). Mediación de conflictos en el plan de mejora: una contribución en el contexto escolar. Campo Abierto. Revista de Educación, 39(2), 181-192.

Proyecto Atlántida. (septiembre, 2003). Escuelas y familias democráticas. Madrid: Proyecto Atlántida.

San Fabián, J. L. (2011). El papel de la organización escolar en el cambio educativo: la inercia de lo establecido. Revista de Educación, 356, 41-60.

Santos Guerra, M. A. (Coord.) (2003). Aprender a convivir en la escuela. Madrid: Akal.

Santos Guerra, M. A. (2009). Escuelas para la democracia. Cultura, organización y dirección de instituciones educativas. Madrid: Wolters Kluwer.

Santos Guerra, M. A. (2015). El valor de la convivencia y el reto de la interculturalidad. Revista Convives, 10, 5-14.

Stake, R. E. (2005). Investigación con estudio de casos (3 ${ }^{\mathrm{a}}$ Ed.). Madrid: Morata.

Taylor, S. J. y Bogdan, R. (1994). Introducción a los métodos cualitativos de investigación ( $2^{\mathrm{a}} \mathrm{ed}$.). Barcelona: Paidós. 
Torrego, J. C. (Coord.) (2006). Modelo integrado de mejora de la convivencia: estrategias de mediación y tratamiento de conflictos. Barcelona: Graó.

Torrego, J. C. (2017). Mediación de conflictos en instituciones educativas. Manual para la formación de mediadores. Madrid: Narcea.

Villareal, O. (2017). ¿Se quiere, se debe y se puede investigar con estudios de casos? Cuadernos de Gestión, 17(1), 147-172.

Weitzman, E. A. (2000). Software and qualitative research. En N. K. Denzin y Y. S. Lincoln (Eds.), Handbook of qualitative research (pp. 803-820). Thousand Oaks: Sage.

Yin, R. (1989). Case Study Research. Design and Methods. London: SAGE.

Zaitegi, N. (2010). La educación en y para la convivencia positiva en España. Revista Iberoamericana sobre Calidad, Eficiencia y Cambio en la Educación, 8(3), 93-132. 